\title{
Preface
}

\section{Waking Up to the Impacts of Sleep Health on Human Performance}

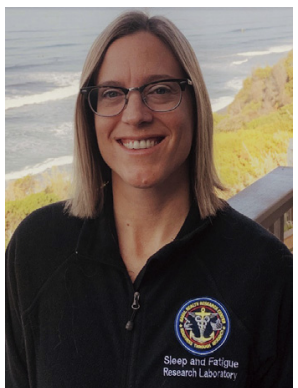

Rachel R. Markwald, PhD

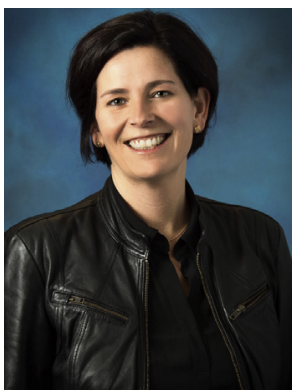

Anne Germain, PhD

Editors

Several decades of research conducted in controlled laboratory settings have illuminated the impact of inadequate sleep quality and/or quantity on neurocognitive performance as well as psychological and physical fitness, readiness, and health. While the magnitude of these effects varies with individual and contextual differences, and across constructs and domains of interest, the findings overwhelmingly indicate a deleterious effect of inadequate sleep on human performance and health. Moving from laboratory assessments into clinical, competitive athletics, and occupational settings, research studies are demonstrating that inadequate sleep has real-world implications on sport and academic performance, recovery from psychological and physical injury/illness, and occupational health and safety. Growing evidence demonstrates that targeting the reestablishment of restorative, sufficient, and consolidated sleep through evidence-based prevention strategies and treatments is accompanied with improvements in performance and clinical outcomes. Novel measurement methods and intervention approaches can play a significant role in translating basic and clinical research findings into effective means of enhancing performance and health across populations. The following articles provide a review of the current research available within these topic areas.
Furthermore, because sleep measurement has expanded beyond the laboratory and clinic into the public health domain, we open this issue with an extensive review on the use and limitations of consumer sleep tracking devices for clinical and human performance research applications. Last, there have been recent advances in the development of neurostimulation technologies for enhancing sleep and performance, and this issue will close with a review on the current research available on these emerging approaches.

Rachel R. Markwald, PhD Sleep and Fatigue Research Laboratory Warfighter Performance Department Naval Health Research Center 140 Sylvester Road San Diego, CA 92101, USA

Anne Germain, $\mathrm{PhD}$ University of Pittsburgh

School of Medicine NOCTEM

218 Oakland Avenue Pittsburgh, PA 15213, USA

E-mail addresses:

Rachel.r.markwald.civ@mail.mil (R.R. Markwald) germax@upmc.edu (A. Germain)

Disclaimer: The views expressed in this editorial are those of the author and do not necessarily reflect the official policy or position of the Department of the Navy, Department of Defense, nor the United States Government. 